University of South Carolina

Scholar Commons

$12-5-2005$

\title{
Top-Down Structure and Device Fabrication using In Situ Nanomachining
}

Xiaodong $\mathrm{Li}$

University of South Carolina - Columbia, lixiao@cec.sc.edu

Xinnan Wang

Qihua Xiong

Peter C. Eklund

Follow this and additional works at: https://scholarcommons.sc.edu/emec_facpub

Part of the Applied Mechanics Commons

\section{Publication Info}

Published in Applied Physics Letters, Volume 87, Issue 23, 2005, pages \#233113-.

(C)Applied Physics Letters 2005, American Institute of Physics.

Li, X., Wang, X., Xiong, Q., \& Eklund, P. C. (5 December 2005). Top-Down Structure and Device Fabrication using In Situ Nanomachining. Applied Physics Letters, 87 (23), http://dx.doi.org/10.1063/1.2139991

This Article is brought to you by the Mechanical Engineering, Department of at Scholar Commons. It has been accepted for inclusion in Faculty Publications by an authorized administrator of Scholar Commons. For more information, please contact digres@mailbox.sc.edu. 


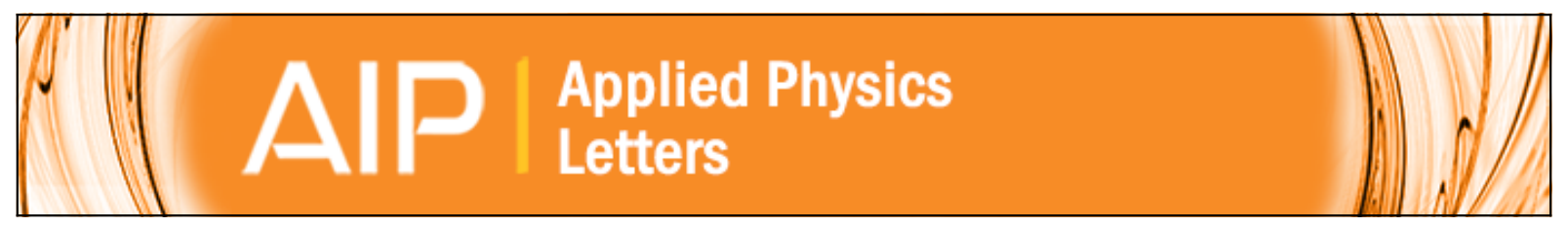

\section{Top-down structure and device fabrication using in situ nanomachining}

Xiaodong Li, Xinnan Wang, Qihua Xiong, and Peter C. Eklund

Citation: Applied Physics Letters 87, 233113 (2005); doi: 10.1063/1.2139991

View online: http://dx.doi.org/10.1063/1.2139991

View Table of Contents: http://scitation.aip.org/content/aip/journal/apl/87/23?ver=pdfcov

Published by the AIP Publishing

\section{Articles you may be interested in}

Enhanced stimulated emission in $\mathrm{ZnO}$ thin films using microdisk top-down structuring

Appl. Phys. Lett. 104, 181104 (2014); 10.1063/1.4875744

In situ study of the growth of $\mathrm{ZnO}$ nanosheets using environmental scanning electron microscope Appl. Phys. Lett. 90, 233104 (2007); 10.1063/1.2746947

Fabrication of metallic nanostructures by atomic force microscopy nanomachining and lift-off process

J. Vac. Sci. Technol. B 22, 2768 (2004); 10.1116/1.1815314

Three-dimensional columnar optical nanostructures fabricated by using lithography-free templating approach Appl. Phys. Lett. 84, 2898 (2004); 10.1063/1.1704858

Nanomachining of mesoscopic electronic devices using an atomic force microscope

Appl. Phys. Lett. 75, 1107 (1999); 10.1063/1.124611

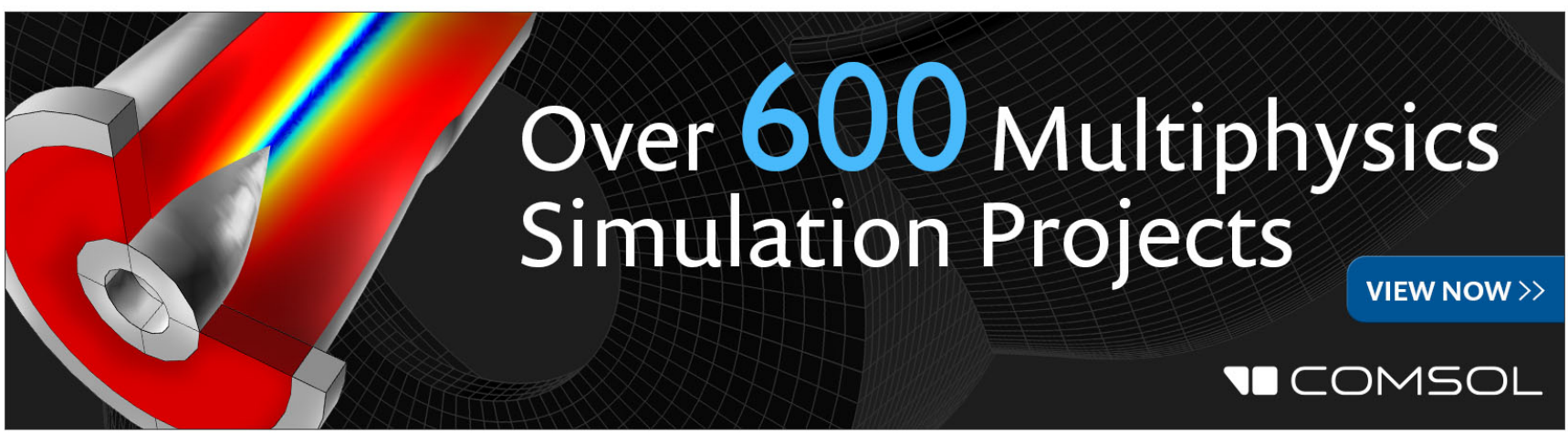




\title{
Top-down structure and device fabrication using in situ nanomachining
}

\author{
Xiaodong $\mathrm{Li}^{\mathrm{a})}$ and Xinnan Wang \\ Department of Mechanical Engineering, University of South Carolina, 300 Main Street, Columbia, \\ South Carolina 29208 \\ Qihua Xiong and Peter C. Eklund \\ Department of Physics, Department of Materials Science and Engineering, Pennsylvania State University, \\ University Park, Pennsylvania 16802
}

(Received 12 September 2005; accepted 24 October 2005; published online 1 December 2005)

\begin{abstract}
We demonstrate the potential of an alternative tool for the fabrication of nanoscale structures and devices. A nanoindenter integrated with an atomic force microscope is shown to be a powerful machine tool for cutting precise length nanowires or nanobelts and for manipulating the shortened wires. We also demonstrate its utility in cutting grooves and fabricating dents (or periodic arrays of dents) in ZnS nanobelts. This approach permits the direct mechanical machining of nanodevices that are supported on a substrate without the inherent complications of e beam or photolithography.

(C) 2005 American Institute of Physics. [DOI: 10.1063/1.2139991]
\end{abstract}

In this letter, we present the results of attempts to use nanomachining to directly create nanoscale structures/ patterns on individual nanofilaments or cut them to prescribed length. Our approach to top-down device fabrication represents a versatile way to create electronic and bioengineering devices, and at the same time, avoids some of the complications inherent in conventional e beam or photolithography. The nanomachining is carried out under ambient conditions using an atomic force microscope (AFM) with a triangular-shaped diamond tip mounted on a stiff stainless steel cantilever.

We demonstrate the capabilities of machining nanoscale objects using $\mathrm{ZnS}$ nanobelts as the raw stock. These nanobelts were grown elsewhere ${ }^{1,2}$ and then deposited from an ethanol suspension onto a $\mathrm{SiO}_{2} / \mathrm{Si}$ substrate for machining. Nanobelts are a convenient starting geometry to demonstrate this approach to device fabrication. Also, $\mathrm{ZnS}$ is an important direct band gap semiconductor $(\sim 3.7 \mathrm{eV})$ that has attracted considerable attention due to its potential application in flatpanel displays, electroluminescent devices, infrared windows, sensors, and lasers. ${ }^{3,4}$ Recently, one-dimensional (1D) $\mathrm{ZnS}$ nanostructures, such as nanobelts and nanowires, have shown a great promise as functional and structural nanobuilding blocks in electronics, optoelectronics, and lasers. ${ }^{1,2,5,6}$

$\mathrm{ZnS}$ nanobelts with the wurtzite structure used in this study were synthesized using the pulsed laser vaporization method, as described in Refs. 1 and 2. Nearly square cross section $\mathrm{ZnS}$ nanobelts of several tens to hundreds of nanometers in width (or height) and several microns in length were first dispersed into ethanol by ultrasonication. Then a few drops of this suspension were deposited either onto lacey carbon copper grids for transmission electron microscopy (TEM, JEOL 2010F), or onto a $\mathrm{SiO}_{2} / \mathrm{Si}$ wafer for scanning electronic microscopy, atomic force microscopy (AFM, Veeco, Dimension 3100), and nanomachining using a Hysitron TriboScope nanoindenter.

\footnotetext{
a) Author to whom correspondence should be addressed; electronic mail:
} lixiao@engr.sc.edu; www.me.sc.edu/research/nano
A Hysitron TriboScope nanoindenter, in conjunction with the Veeco Dimension 3100 AFM, was used to perform imaging and nanoindentation tests for mechanical property measurements. ${ }^{7}$ The same tool was used for in situ nanomachining of the nanobelts supported on the substrate. The nanoindenter monitors and records the load and displacement of the three-sided pyramidal Berkovich diamond tip during indentation. It has a force resolution of $\sim 1 \mathrm{nN}$ and a displacement resolution of about $0.2 \mathrm{~nm} .{ }^{8,9}$

In Fig. 1(a) we display a TEM bright-field image of $\mathrm{ZnS}$ nanobelts showing their geometrical shape. Detailed examination of the nanobelts along their length reveals that they are very uniform in width and thickness. Figure 1(b) shows a TEM cross-sectional image of a single nanobelt that has an approximately square cross section. With our technique, we can use the AFM to predetermine the cross section of the nanobelt to which we will apply our nanomachining process. This is a second important advantage of our approach, i.e., the ability to select a particular dimension device from which to fabricate a sensor or transistor. The high resolution TEM image [Fig. 1(c)] shows a lattice image of a selected nanobelt; the lattice plains are seen to be very well organized along the growth direction. Many such images reveal that the $\mathrm{ZnS}$ nanobelts are often a single crystal with a [001] growth direction. They appear to be dislocation free, as also indicated by the selective-area diffraction pattern [inset to Fig. 1(c)]. The measured distance between the lattice fringes is $3.19 \AA$ for the (001) planes, consistent with the $d$ spacing value listed in x-ray diffraction powder diffraction files (10434) for bulk w-ZnS. ${ }^{10}$ Data such as the AFM images [Fig. 1(d)] and section analysis [Fig.1(e)] and the TEM cross section suggest that the majority of the nanobelts have nearly a square cross section.

By using a high indentation load, a nanobelt can be cut into the length as needed. A $10-\mu \mathrm{m}$-long $\mathrm{ZnS}$ nanobelt was cut into ten pieces and then imaged in situ using the same indenter tip. Figure 2(a) shows an AFM image of a $\sim 1-\mu \mathrm{m}$-long nanobelt that has been cut from the longer one. The corresponding indentation load-displacement curve in the region of elastic-plastic deformation leading up to and including fracture is shown in Fig. 2(c) (dent formation). 


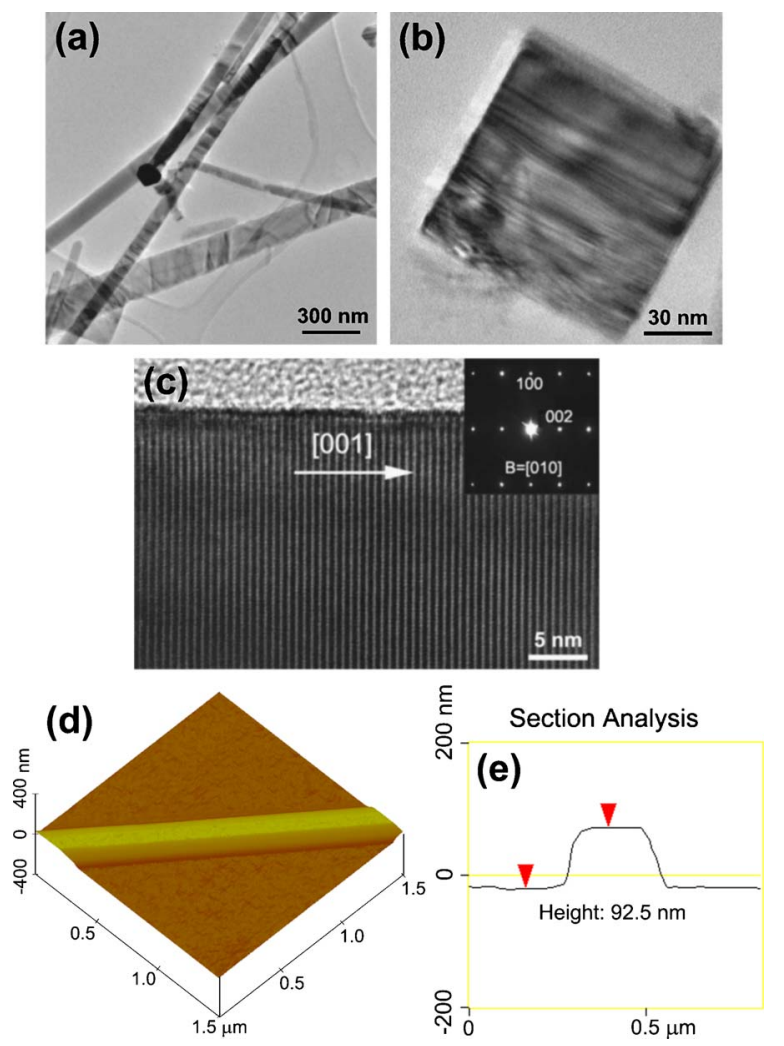

FIG. 1. (Color online) (a) Bright-field TEM image of ZnS nanobelts. (b) TEM image of the cross section of a $\mathrm{ZnS}$ nanobelt. (c) High resolution TEM image of a $\mathrm{ZnS}$ nanobelt growing along a [001] direction. The inset shows a selective-area electron diffraction pattern from the same nanobelt. (d) Threedimensional AFM image of a single $\mathrm{ZnS}$ nanobelt. (e) Cross-sectional profile shows the thickness of the nanobelt in (d) is $93 \mathrm{~nm}$.

Interestingly, we see a significant change in the Young's modulus at the nanoscale-from $75 \mathrm{GPa}$ for bulk w-ZnS down to $35.9 \mathrm{GPa}$ for the same crystal structure nanobelts. This significant difference in mechanical properties may be important to the ease of machining at the nanoscale and is a third important consideration in our approach. A step in the loading curve was observed at $144 \mu \mathrm{N}$ load [Fig. 2(c)], which resulted from the fracture of the nanobelt. Before fracture, the nanobelt experienced elastic/plastic deformation, as can be seen from the loading curve in Fig. 2(c). Figure 2(d) schematically shows how to estimate the energy release during the nanoindentation from the load-displacement curve as shown in Fig. 2(c). The energy release $\Delta \mathrm{E}$ in cutting can be estimated from Fig. 2(c) to be $\Delta E=5.72 \times 10^{-13} \mathrm{~J}$ or 3.575 $\times 10^{6} \mathrm{eV}$, corresponding to the fracture of about one million bonds.

To date, virtually all nanoelectronic devices have been made by adding contacts and electrical leads to nanowires and nanotubes and using back- or proximal gating to control the current through the devices. ${ }^{11-16}$ Prototype devices such as transistors, ${ }^{11}$ gates, ${ }^{12}$ and others devices ${ }^{13-16}$ have been fabricated from uniform cross section wires or ribbons grown elsewhere or grown in place on the substrate. An alternative approach to these uniform nanowire devices is the fabrication of a different class of devices based on nonuniform physical properties or cross section that can be achieved by nanomachining, i.e., the introduction of a large scale dent, or a periodic series of dents, with each dent acting as a position for the fabrication of a "quantum dot." Similarly, one can imagine introducing a machined groove along the nanobelt

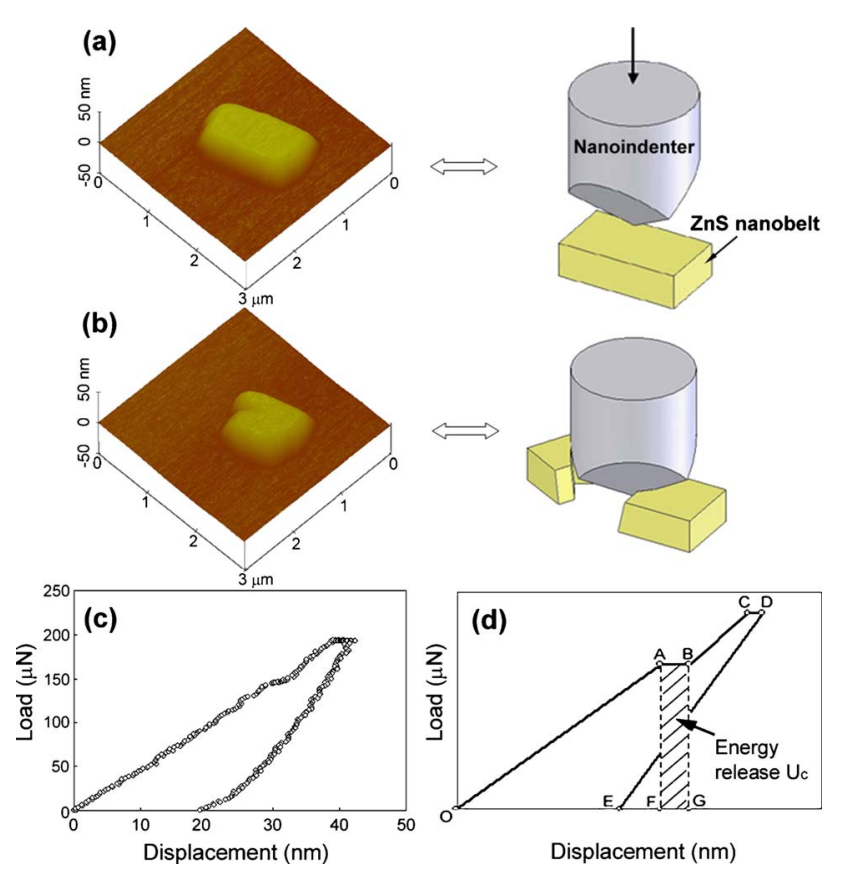

FIG. 2. (Color online) (a) Three-dimensional AFM image and schematic of a nanobelt before cutting with diamond indenter tip. (b) Three-dimensional AFM image and schematic of nanoindentation cutting a nanobelt. (c) Nanoindentation load-displacement curve and (d) schematic loaddisplacement curve, showing a step during loading cycle and associated energy release.

by dragging the indenter/AFM tip down the belt axis. Our intent in this work is to demonstrate the capability of fabricating these interesting classes of electronic devices.

In this study, an array of nanoscale pyramidal indentations of different sizes and depths was also made on a single $\mathrm{ZnS}$ nanobelt using an AFM diamond tip. A series of impressions along the nanobelt axis can be seen in the AFM image of Fig. 3(a). A z scan of these impressions is shown in Fig. 3(b). Indents of different shapes can be obtained by using nanoindenters with different tip shapes, such as cubic, conical and spherical geometries. Such nanoscale cavities can be used as quantum wells for nanoelectronics (quantum dots), but they also might be used for cells for molecule electronics and drug delivery, slots for integration of the nanobuilding blocks into functional nanostructures and nanodevices, and scattering defects.

Furthermore, nanochannels are essential components in nanofluidic systems. However, fabricating nanochannels directly on a nanobuilding block, such as a nanobelt, has not yet been achieved and offer a possibility for applications in a complex drug delivery system, lubricating functionality, and nanomechanical switches (nanogears). To machine such a channel, we have found that a stiffer cantilever (with a diamond tip) is necessary. By dragging the tip along the nanobelt axis with constant force, a channel can be created, as shown in Fig. 3(c). Interestingly, post in situ imaging of the channel in a $\mathrm{ZnS}$ nanobelt [Fig. 3(c)] also reveals no debris (particles) along the scratch path or at the end of the channel.

Using our nanomachining approach, multifunctional nanofluidic pumps and nanofluidic sensors may be realized on a single nanobelt. Such combination of tunable nanochannels and nanoslots might be expected to impact molecular 

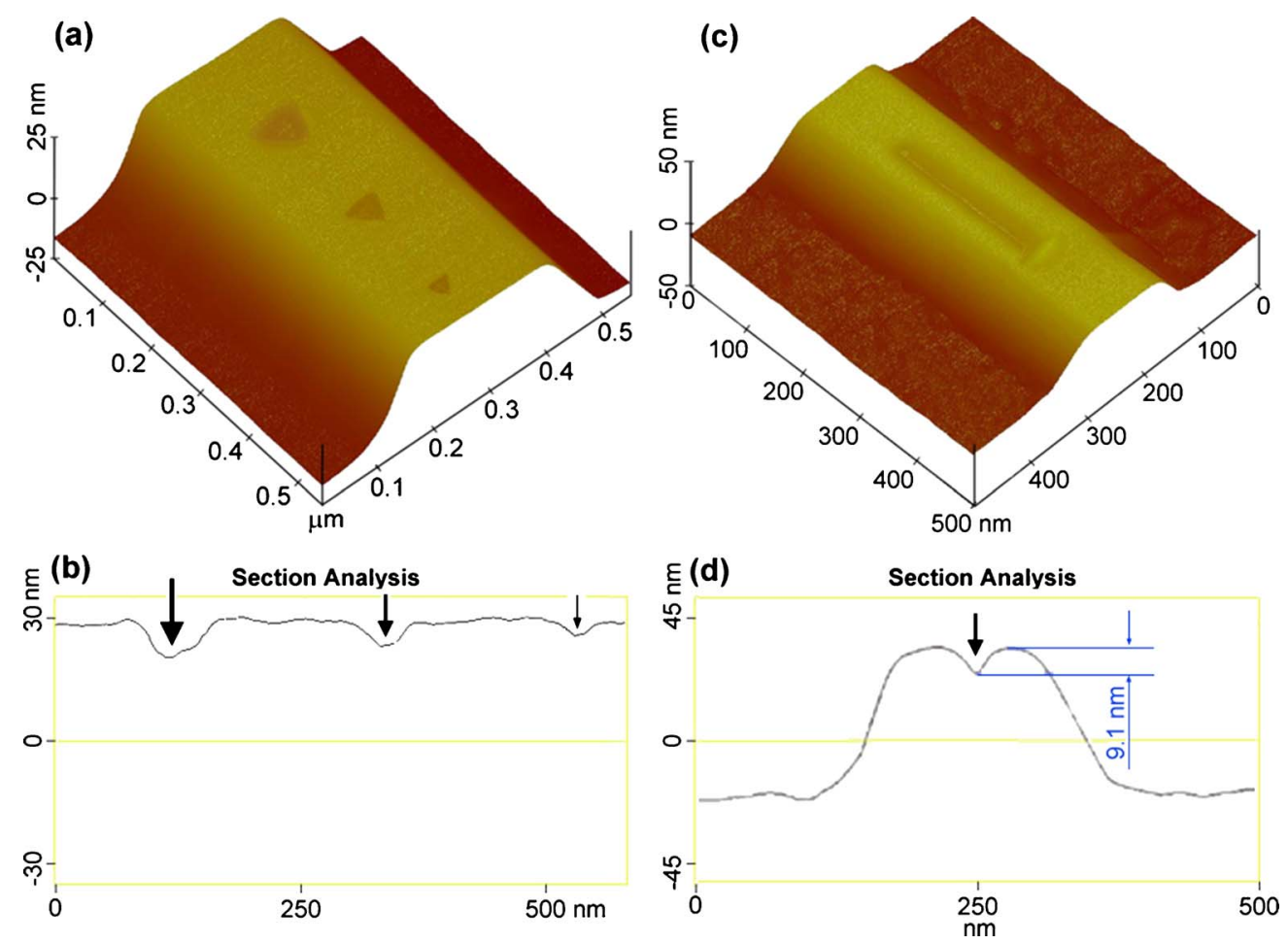

FIG. 3. (Color online) (a) An AFM image of an array of nanoindents fabricated on the top surface of a ZnS nanobelt. The indentations are created with a diamond AFM tip at loads of 18.9, 15.2 and $11.4 \mu \mathrm{N}$, respectively, and imaged with the same tip in situ. (b) Cross-sectional view ( $z$ scan) of indentation depths of successively deeper dents. (c) AFM image of a nanochannel fabricated by dragging the AFM diamond dip along the nanobelt. (d) Cross-sectional ( $z$ scan) profile of the nanochannel.

electronics and many other disciplines such as biology, chemistry, medicine, and materials. High-volume batch processing may be realized by machining self-assembled, wellaligned and controlled-length 1D nanostructures using multiple probes, as shown schematically in Fig. 4.

Financial support for this study was provided by the National Science Foundation (Grant No. EPS-0296165), the

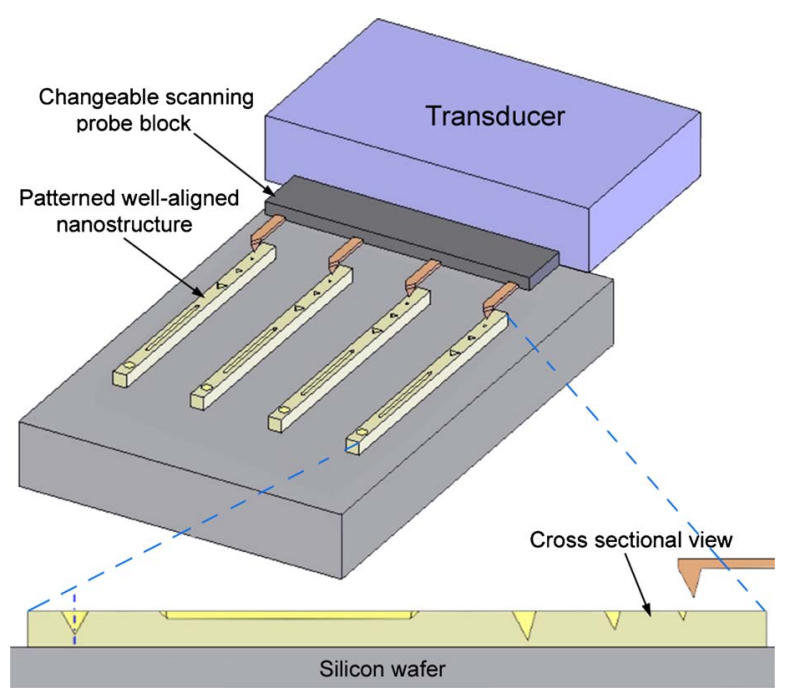

FIG. 4. (Color online) Schematic illustration of possible batch mechanical machining approach to self-assembled and well-aligned 1D nanostructures.
ACS Petroleum Research Fund (ACS PRF No. 40450AC10), the South Carolina Space Grant Consortium-NASA, and the University of South Carolina NanoCenter Seed Grant. P.C.E. would like to acknowledge the support from NSF-NIRT (Nanotechnology and Interdisciplinary Research Initiative), Grant No. DMR-0304178.

${ }^{1}$ Q. Xiong, G. Chen, J. D. Acord, X. Liu, J. J. Zengel, H. R. Gutierrez, J. M. Redwing, L. C. Lew Yan Voon, B. Lassen, and P. C. Eklund, Nano Lett. 4, 1663 (2004).

${ }^{2}$ Q. Xiong, J. Wang, O. Reese, L. C. Lew Yan Voon, and P. C. Eklund, Nano Lett. 4, 1991 (2004).

${ }^{3}$ C. Falcony, M. Garcia, A. Ortiz, and J. C. Alonso, J. Appl. Phys. 72, 1525 (1992).

${ }^{4}$ T. V. Prevenslik, J. Lumin. 87-89, 1210 (2000).

${ }^{5}$ Y. Jiang, X. M. Meng, J. Liu, Z. Y. Xie, C. S. Lee, and S. T. Lee, Adv. Mater. (Weinheim, Ger.) 15, 323 (2003).

${ }^{6}$ Y. C. Zhu, Y. Bando, and D. F. Xue, Appl. Phys. Lett. 82, 1769 (2003).

${ }^{7}$ X. Li, X. Wang, Q. Xiong, and P. C. Eklund, Nano Lett. 5, 1982 (2005).

${ }^{8} \mathrm{X}$. Li and B. Bhushan, Mater. Charact. 48, 11 (2002).

${ }^{9}$ B. Bhushan and X. Li, Int. Mater. Rev. 48, 125 (2003).

${ }^{10}$ Powder Diffraction File, Inorganic Volume, Swarthmore, PA.: JCPDS International Centre for Diffraction Data, 1960-1987.

${ }^{11}$ D. L. Feldheim and C. D. Keating, Chem. Soc. Rev. 27, 1 (1998).

${ }^{12} \mathrm{H}$. T. Ng, J. Han, T. Yamada, P. Nguyen, Y. P. Chen, and M. Meyyappan, Nano Lett. 4, 1247 (2004).

${ }^{13}$ X. Duan, Y. Huang, Y. Cui, and C. M. Lieber, in Molecular Nanoelectron$i c s$, edited by M. A. Reed and T. Lee (American Scientific, Stevenson Ranch, CA, 2003), pp. 199-227.

${ }^{14}$ G. Maruccio, R. Cingolani, and R. Rinaldi, J. Mater. Chem. 14, 542 (2004).

${ }^{15}$ S. Tan, Z. Tang, X. Liang, and N. A. Kotov, Nano Lett. 4, 1637 (2004).

${ }^{16}$ M. W. Dashiell, R. T. Troeger, S. L. Rommel, T. N. Adam, P. R. Berger, C. Guedj, J. Kolodzey, A. C. Seabaugh, and R. Lake, IEEE Trans. Electron Devices 47, 1707 (2000). 